\title{
Generation of a High-Visibility Four-Photon Entangled State and Realization of a Four-Party Quantum Communication Complexity Scenario
}

\author{
Jin-Shi Xu, Chuan-Feng Li*, and Guang-Can Guo \\ Key Laboratory of Quantum Information, University of Science and Technology of China, \\ CAS, Hefei, 230026, People's Republic of China
}

(Dated: April 17, 2018)

\begin{abstract}
We obtain a four-photon polarization-entangled state with a visibility as high as $(95.35 \pm 0.45) \%$ directly from a single down-conversion source. A success probability of $(81.54 \pm 1.38) \%$ is observed by applying this entangled state to realize a four-party quantum communication complexity scenario (QCCS), which comfortably surpass the classical limit of 50\%. As a comparison, two EinsteinPodolsky-Rosen (EPR) pairs are shown to implement the scenario with a success probability of $(73.89 \pm 1.33) \%$. This four-photon state can be used to fulfill decoherence-free quantum information processing and other advanced quantum communication schemes.
\end{abstract}

PACS numbers: 03.67.Mn, 03.67.Hk, 42.65.Lm, 42.50.Ar

Entanglement is one of the most important and interesting characteristic of quantum mechanics. Entangled states of two or more particles not only play a central role in the discussion of quantum mechanics versus local realism [1], but also form the basis of nearly all quantum information protocols, including quantum cryptography [2], quantum computation [3], dense coding [4], teleportation [5] and quantum communication complexity [6, 7, [] ].

Many experiments employing type-II spontaneous parametric down-conversion (SPDC) process have been reported to realize multiphoton entangled states, including a four-photon Greenberger-Horne-Zeilinger (GHZ) state with a visibility of $(79 \pm 6) \%[\underline{9}$, a four-photon decoherence-free state of visibility $(79.3 \pm 1.4) \%[10]$ and a four-photon cluster state with a fidelity of $(74.1 \pm 1.3) \%$ 11. Yet, in those schemes interference occurs pairwise between processes where the photon pair is created at distances $\pm x$ from the middle of the crystal [12], which may limit the purity of the state.

In this letter, we show that a polarization-entangled state observed behind a single pulsed type-I SPDC source can reach a visibility as high as $(95.35 \pm 0.45) \%$. We use this state to realize a four-party quantum communication complexity scenario (QCCS) with a success probability of $(81.54 \pm 1.38) \%$, which is much higher than the classical limit of $50 \%$. According to C. Brukner et al. [13] this is equal to show that our state violate a kind of Bell's inequality.

There is a reasonable probability of simultaneously producing four photons in a single strong pulsed SPDC source. In our experiment, we use two identically cut type-I beta-barium-borate $(\mathrm{BBO})$ crystals $(8.0 \times 8.0 \times 0.6$ $\left.\mathrm{mm}, \theta_{p m}=30.35^{\circ}\right)$ with their optic axes aligned in mutually perpendicular planes [14]. Frequency doubled ultraviolet (UV) pluses (390 nm center wavelength, $\sim 200 \mathrm{fs}$ pulse duration, $76 \mathrm{MHz}$ repetition rate, $\sim 500 \mathrm{~mW}$ average power) from a mode-locked Ti:sapphire laser is used

\footnotetext{
*email: cfli@ustc.edu.cn
}

to pass through the two-crystal geometry BBO. Behind two 50-50 beam splitters, the four photons with distinct spatial mode are coupled into single mode optical fibers (Fig. 1).

According to Schrödinger equation, the four-photon state can be obtained as

$$
\begin{aligned}
\left|\Psi^{4}\right\rangle & =\left(a_{H}^{\dagger} b_{H}^{\dagger}+a_{V}^{\dagger} b_{V}^{\dagger}\right)^{2}|0\rangle \\
& =\left(a_{H}^{\dagger 2} b_{H}^{\dagger 2}+a_{V}^{\dagger 2} b_{V}^{\dagger 2}+2 a_{H}^{\dagger} b_{H}^{\dagger} a_{V}^{\dagger} b_{V}^{\dagger}\right)|0\rangle,
\end{aligned}
$$

where $a_{H}^{\dagger}$ is the creation operator of a photon with horizontal polarization in mode $a$, etc.

For simplicity, we assume that at the beam splitters $a$ is transformed into $\frac{1}{\sqrt{2}}(c+e)$ and $b$ into $\frac{1}{\sqrt{2}}(d+f)$ [15], where $c, d$ and $e, f$ denote the transmitted and reflected modes, respectively. We then expand Eq. (1) and keep only those terms which lead to four-photon coincidence behind the two beam splitters, i.e., only those terms for which there is one photon in each of the modes. As a result, this four-photon state can be written as

$$
\begin{aligned}
\left|\Psi^{4}\right\rangle= & |H H H H\rangle_{c d e f}+|V V V V\rangle_{c d e f} \\
& +\frac{1}{2}\left(|H H V V\rangle_{c d e f}+|H V V H\rangle_{c d e f}\right. \\
& \left.+|V H H V\rangle_{c d e f}+|V V H H\rangle_{c d e f}\right),
\end{aligned}
$$

where $|H H H H\rangle_{\text {cdef }}$ denotes a $H$ polarized photon in each mode of $c, d, e$ and $f$, etc.

This state can be seen as the superposition of a four-photon GHZ state and a product of two EinsteinPodolsky-Rosen (EPR) pairs (normalized)

$$
\left|\Psi^{4}\right\rangle=\sqrt{\frac{2}{3}}|G H Z\rangle_{c e d f}+\sqrt{\frac{1}{3}}|E P R\rangle_{c e} \otimes|E P R\rangle_{d f},
$$

where $|G H Z\rangle=\frac{1}{\sqrt{2}}(|H H H H\rangle+|V V V V\rangle)$ is the GHZ state, $|E P R\rangle=\frac{1}{\sqrt{2}}(|H V\rangle+|V H\rangle)$ is the EPR state $\left|\Psi^{+}\right\rangle$.

Many efforts have been made to keep our experimental system stable for several days. An air conditioner is used to keep the room temperature to the order of 

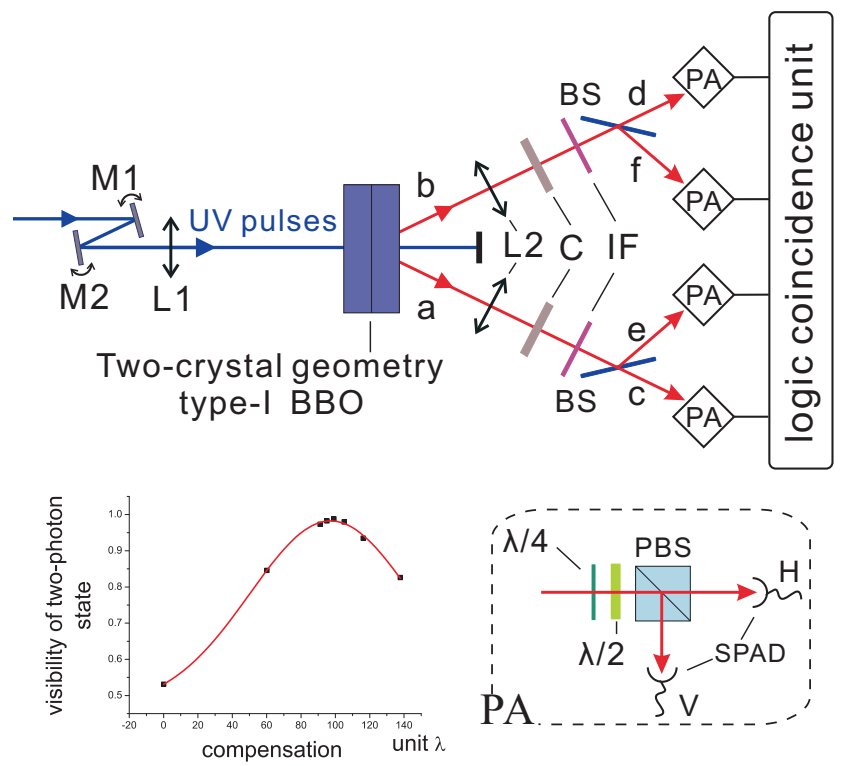

FIG. 1: (Color on line). Experimental setup. The UV pulse is focused by a convex lens with a focal length of $50 \mathrm{~cm}$ (L1) and the direction of the UV beam is controlled by two mirrors M1 and M2. The four photons are emitted into two spatial modes $a$ and $b$. After collected by a convex lens with a focal length of $30 \mathrm{~cm}$ (L2) in each mode, the four photons pass through quartz plates $(\mathrm{C})$ to compensate the birefringence in BBO. Then, they are distributed into the four modes $c, d, e$ and $f$ by two $50-50$ beam splitters (BS) behind interference filters (IF, $\Delta \lambda=3 \mathrm{~nm}, \lambda=780 \mathrm{~nm}$ ). In order to analyze the four-photon state and to realize the QCCS, polarization analysis (PA) in various bases is performed for each mode using quarter wave plates $(\lambda / 4)$ and half wave plates $(\lambda / 2)$ in front of polarizing beam splitters (PBS) and single photon avalanche detectors (SPAD). The inset shows the visibility of two-photon entangled state versus compensation (100 mW pump). The solid line is a Gaussian function fitting (unit $\lambda=780 \mathrm{~nm})$.

$\pm 1^{\circ} \mathrm{C}$. To avoid damage to the second harmonic generation $\mathrm{BBO}$ and the SPDC BBO, we pump $\mathrm{N}_{2}$ around them. Moreover, by using a motion controller system (Newport, NSC200) to tilt two mirrors M1 and M2 (in Fig. 1) with the feedback of two charge coupled devices (not shown) and twofold coincidences of two paths (such as modes $c$ and $d$ ), we manage to maintain the position of the pump beam.

To obtain the high-purity four-photon entangled state, the birefringence between horizontal and vertical photons in the two-crystal geometry BBO has been compensated with quartz plates. The inset of Fig. 1 shows that the coherence between horizontal and vertical photons is recovered perfectly while the compensation of optical path difference is about $99.1 \lambda$.

Fig. 2(a) and (b) show the 16 possible fourfold coincidence probabilities for detecting one photon in each mode with the four polarization analyzers oriented along $H / V$ basis and $+/-$ basis $\left( \pm 45^{\circ}\right.$ linear polarizations, i.e.,
$\left.\frac{1}{\sqrt{2}}(H \pm V)\right)$, respectively [10]. The integration time is 3 hours per column. One can find two types of coincidences, the GHZ part, and the fourfold coincidences due to the EPR pairs with average rates lower by a factor of 4 , which is in very good agreement with the state in Eq. (3).

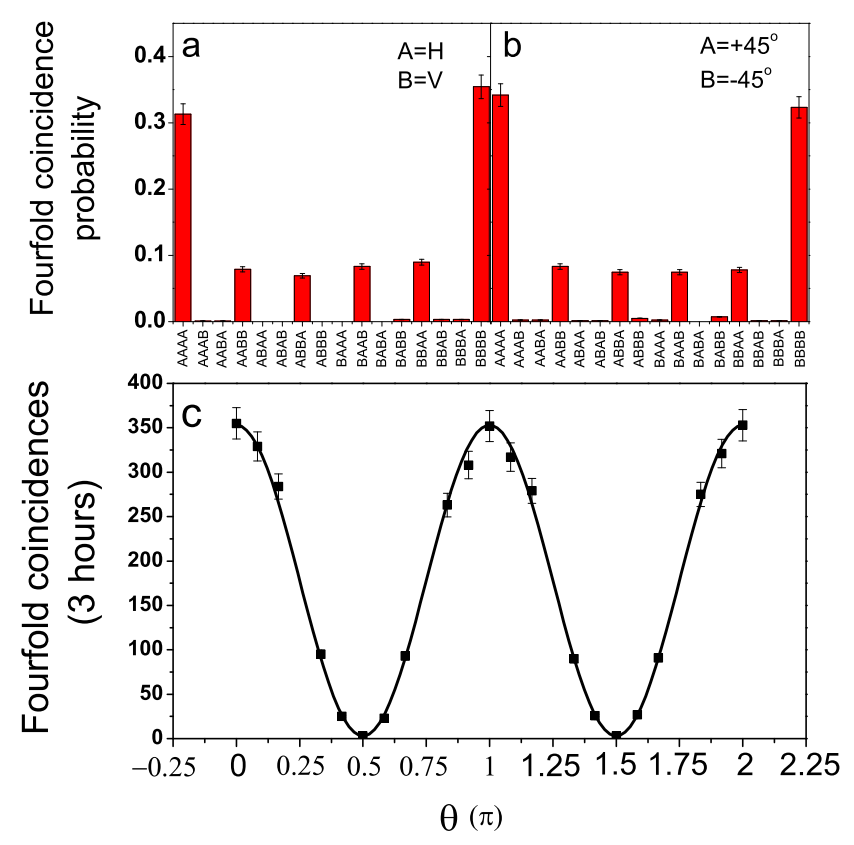

FIG. 2: (Color on line). Fourfold coincidence probabilities corresponding to different measurement basis settings. (a) the $H / V$ basis; (b) the $+/-$ basis. (c) four-photon interference curve. We vary the detection basis in mode $f$, while keeping mode $c, d, e$ in the $+\left(+45^{\circ}\right)$ basis. $\theta$ represent the angle between the linear polarization detection basis and the + basis in mode $f$. The solid line shows a sinusoidal fit to the experimental results with a visibility of $(98.45 \pm 0.15) \%$.

Fig. 2(c) shows one of the four-photon interference curves of the entangled state. More strictly, we may use the correlation function to characterize the entangled state. The experimental value of the correlation function is obtained from the 16 four-photon coincidence rates with [10]

$$
\begin{aligned}
& E\left(\phi_{c}, \phi_{e}, \phi_{d}, \phi_{f}\right)= \sum_{\substack{l_{c}, l_{e}, l_{d}, l_{f}= \pm 1 \\
\times}} l_{c}, l_{e}, l_{d}, l_{f} \\
& \times P_{l_{c}, l_{e}, l_{d}, l_{f}}\left(\phi_{c}, \phi_{e}, \phi_{d}, \phi_{f}\right),
\end{aligned}
$$

where $l_{x}, \phi_{x}$ are corresponding to the eigenvectors $\left|l_{x}, \phi_{x}\right\rangle=\frac{1}{\sqrt{2}}\left(|V\rangle_{x}+l_{x} e^{-i \phi_{x}}|H\rangle_{x}\right)$ with eigenvalues $l_{x}=$ \pm 1 for polarization measurements performed by the observation stations in the four modes $(x=c, e, d, f)$ and $P_{l_{c}, l_{e}, l_{d} l_{f}}$ are the four-photon probabilities. Theoretically [15], when $\phi_{c}=\phi_{e}=\phi_{d}=\phi_{f}=0$, the correlation function reaches its maximal value, which is equal to the visibility of the curve of $E$ versus one of the angles, such as $\phi_{c}$, with other angles $\phi_{e}=\phi_{d}=\phi_{f}=0$. From 
the data of Fig. 2(b), we obtain $V=(95.35 \pm 0.45) \%$, compared to the theoretical result $V=100 \%$ for a pure state.

During the experiment, different setup is attempted to achieve better result. For example, if the lens with a focal length of $50 \mathrm{~cm}$ is replaced by a lens of $30 \mathrm{~cm}$ to focus the pump pulse onto the crystal, the four-photon coincidences will be 4 times brighter, however the fourphoton visibility will decrease to about $80 \%$.

Next, we use this entangled state to realize a four-party QCCS.

Suppose there are four parties A, B, C and D receiving $X, Y, Z$ and $K$, respectively, where $X, Y, Z, K \in U \in$ $\{0,1\}^{2}$, and they are promised that

$$
(X+Y+Z+K) \bmod 2=0 .
$$

The common goal is for each party to get the correct value of the Boolean function

$$
F(X, Y, Z, K)=\frac{1}{2}[(X+Y+Z+K) \bmod 4] .
$$

$X, Y, Z$ and $K$ can be represented in binary notation as $x_{1} x_{0}, y_{1} y_{0}, z_{1} z_{0}$ and $k_{1} k_{0}$. According to Eq. (5) $x_{0} y_{0} z_{0} k_{0}$ is one of the eight combinations 0000, 0011, 0101, 0110, 1001, 1010, 1100, 1111.

We then rewrite Eq. (6) as

$$
F=x_{1} \oplus y_{1} \oplus z_{1} \oplus k_{1} \oplus F_{0}\left(x_{0}, y_{0}, z_{0}, k_{0}\right),
$$

where

$$
F_{0}\left(x_{0}, y_{0}, z_{0}, k_{0}\right)=\frac{1}{2}\left[\left(x_{0}+y_{0}+z_{0}+k_{0}\right) \bmod 4\right] .
$$

As a result, if $x_{0} y_{0} z_{0} k_{0}=0000$ or $1111, F_{0}=0$, else, $F_{0}=1$.

Obviously, if these four parties are restricted to broadcast one bit respectively, they have $50 \%$ probability to get the correct value of $F$ in classical situation [8]. On the other hand, if they share the four-photon entangled state we have prepared initially, the probability they get the correct value of $F$ can reach $83.33 \%$, as shown below.

Each of the four parties A, B, C and D share one photon of the state

$$
\begin{aligned}
\left|\Psi^{4}\right\rangle= & \frac{1}{\sqrt{3}}(|0000\rangle+|1111\rangle) \\
& +\frac{1}{2 \sqrt{3}}(|0011\rangle+|1001\rangle+|0110\rangle+|1100\rangle),
\end{aligned}
$$

where 0 and 1 represents $H$ and $V$ in Eq. (2), respectively. (we have omitted the subscripts $c, d, e, f$ for simplicity).

If $x_{0}\left(y_{0}, z_{0}, k_{0}\right)=0$, then $\mathrm{A}(\mathrm{B}, \mathrm{C}, \mathrm{D})$ applies rotation

$$
R(x)=\frac{1}{\sqrt{2}}\left(\begin{array}{cc}
1 & 1 \\
1 & -1
\end{array}\right)
$$

on his own photon with half wave plate and quarter wave plate; if $x_{0}\left(y_{0}, z_{0}, k_{0}\right)=1$, then $\mathrm{A}(\mathrm{B}, \mathrm{C}, \mathrm{D})$ applies rotation

$$
R(y)=\frac{1}{\sqrt{2}}\left(\begin{array}{cc}
1 & i \\
i & 1
\end{array}\right)
$$

on his own photon. Then each of the four parties measures the photon under $0 / 1(H / V)$ basis and get the result of $a, b, c, d$. Due to the entanglement of the state they share initially, A, B, C, D only have to broadcast the four bits $x_{1} \oplus a, y_{1} \oplus b, z_{1} \oplus c, k_{1} \oplus d$, respectively. Then they have on average $83.33 \%$ probability to get the correct value of $F$ as

$$
F=x_{1} \oplus a \oplus y_{1} \oplus b \oplus z_{1} \oplus c \oplus k_{1} \oplus d,
$$

that is

$$
F_{0}=a \oplus b \oplus c \oplus d .
$$

For example, in the case $x_{0} y_{0} z_{0} k_{0}=0000$, local rotations $R(x) \otimes R(x) \otimes R(x) \otimes R(x)$ do not change the four-photon state, i.e., $\left|\Psi^{4}\right\rangle^{\prime}=\left|\Psi^{4}\right\rangle$, where $\left|\Psi^{4}\right\rangle^{\prime}$ is the state after local rotations. Consequently, the success probability is $100 \%$. The remaining cases can be similarly analyzed, as shown in table I.

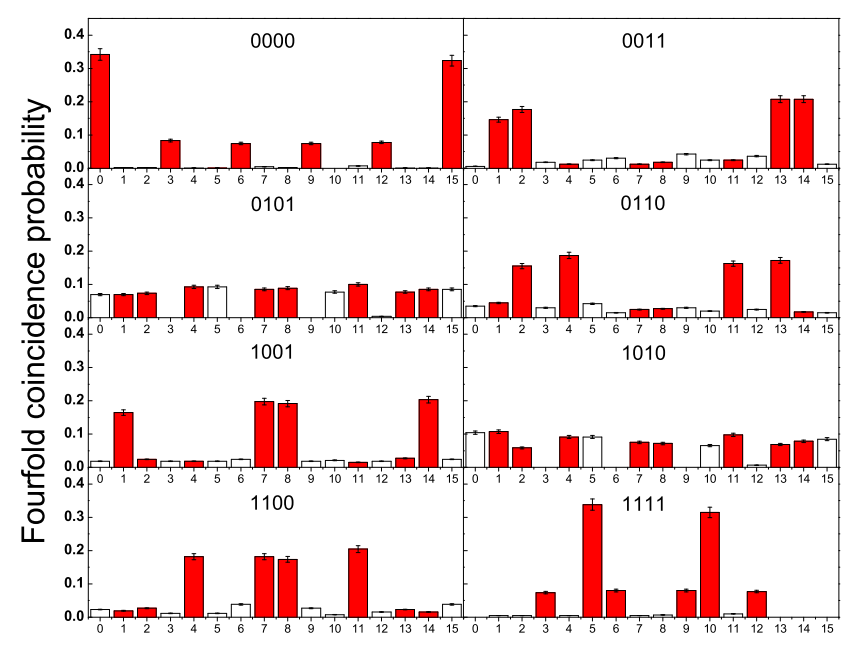

FIG. 3: (Color on line). Fourfold coincidence probabilities for the four-party QCCS. Each frame represents a kind of combination of $x_{0} y_{0} z_{0} k_{0}$, denoted by 0000,0011 , etc. The $x$ axis of each frame $(0,1 \cdots 15)$ represents the sixteen different $0 / 1$ $(H / V)$ basis settings in binary representation, e.g., $9=1001$. The filled and unfilled columns denote the probabilities of getting the correct and wrong value of $F$, respectively.

Fig. 3 illustrates the experimental result in detail. It is shown that the average probability for the four parities to get the correct value of $F$ in our experiment is $(81.54 \pm$ $1.38) \%$, which greatly surpass the classical limit of $50 \%$. This result prove that the state we have prepared violate a kind of Bell's inequality [13].

To illustrate that there is genuine four-photon entanglement in the state we have prepared, we further consider another case, where $(\mathrm{A}, \mathrm{B})$ and $(\mathrm{C}, \mathrm{D})$ share two EPR states $\left|\Phi^{+}\right\rangle=\frac{1}{\sqrt{2}}(|H H\rangle+|V V\rangle)=\frac{1}{\sqrt{2}}(|00\rangle+|11\rangle)$, respectively. It can be deduced that the probability for 
TABLE I: The input of $x_{0} y_{0} z_{0} k_{0}$, the corresponding local rotations, the components of $\left|\Psi^{4}\right\rangle^{\prime}$ for successful communication, the result of $F_{0}$, the corresponding theoretical probability (theor. prob.) and experimental probability (expt. prob.) to get the correct value of $F$.

\begin{tabular}{|c|c|c|c|c|c|c|c|c|}
\hline$x_{0} y_{0} z_{0} k_{0}$ & Local rotations & & $\left.\Psi^{4}\right\rangle^{\prime}$ & & & $F_{0}$ & Theor. prob. & Expt. prob. \\
\hline 0000 & $R(x) \otimes R(x) \otimes R(x) \otimes R(x)$ & \multirow{2}{*}{\begin{tabular}{|l|}
$0000\rangle$ \\
$1001\rangle$
\end{tabular}} & \multirow{2}{*}{\begin{tabular}{|l|}
$0011\rangle$ \\
$|1010\rangle$
\end{tabular}} & \multirow{2}{*}{\begin{tabular}{|l|}
$0101\rangle$ \\
$|1100\rangle$
\end{tabular}} & \multirow{2}{*}{\begin{tabular}{|l|}
$0110\rangle$ \\
$|1111\rangle$
\end{tabular}} & 0 & $100 \%$ & $97.68 \% \pm 0.23 \%$ \\
\hline 1111 & $R(y) \otimes R(y) \otimes R(y) \otimes R(y)$ & & & & & 0 & $100 \%$ & $96.32 \% \pm 0.35 \%$ \\
\hline 0011 & $R(x) \otimes R(x) \otimes R(y) \otimes R(y)$ & \multirow{4}{*}{$\begin{array}{l}|0001\rangle \\
|1000\rangle\end{array}$} & \multirow{4}{*}{$\begin{array}{l}|0010\rangle \\
|1011\rangle\end{array}$} & \multirow{4}{*}{$\mid \begin{array}{l}|0100\rangle \\
|1101\rangle\end{array}$} & \multirow{4}{*}{$\begin{array}{l}|0111\rangle \\
|1110\rangle\end{array}$} & 1 & $83.33 \%$ & $80.49 \% \pm 1.57 \%$ \\
\hline 1100 & $R(y) \otimes R(y) \otimes R(x) \otimes R(x)$ & & & & & 1 & $83.33 \%$ & $82.63 \% \pm 1.44 \%$ \\
\hline 0110 & $R(x) \otimes R(y) \otimes R(y) \otimes R(x)$ & & & & & 1 & $83.33 \%$ & $79.06 \% \pm 1.66 \%$ \\
\hline 1001 & $R(y) \otimes R(x) \otimes R(x) \otimes R(y)$ & & & & & 1 & $83.33 \%$ & $84.13 \% \pm 1.34 \%$ \\
\hline 0101 & $R(x) \otimes R(y) \otimes R(x) \otimes R(y)$ & \multirow{2}{*}{$\begin{array}{l}|0001\rangle \\
|1000\rangle\end{array}$} & \multirow{2}{*}{$\begin{array}{l}0010\rangle \\
1011\rangle\end{array}$} & \multirow{2}{*}{$\begin{array}{l}|0100\rangle \\
|1101\rangle\end{array}$} & \multirow{2}{*}{\begin{tabular}{|l|}
$0111\rangle$ \\
$|1110\rangle$
\end{tabular}} & 1 & $66.67 \%$ & $67.18 \% \pm 2.20 \%$ \\
\hline 1010 & $R(y) \otimes R(x) \otimes R(y) \otimes R(x)$ & & & & & 1 & $66.67 \%$ & $64.82 \% \pm 2.28 \%$ \\
\hline
\end{tabular}

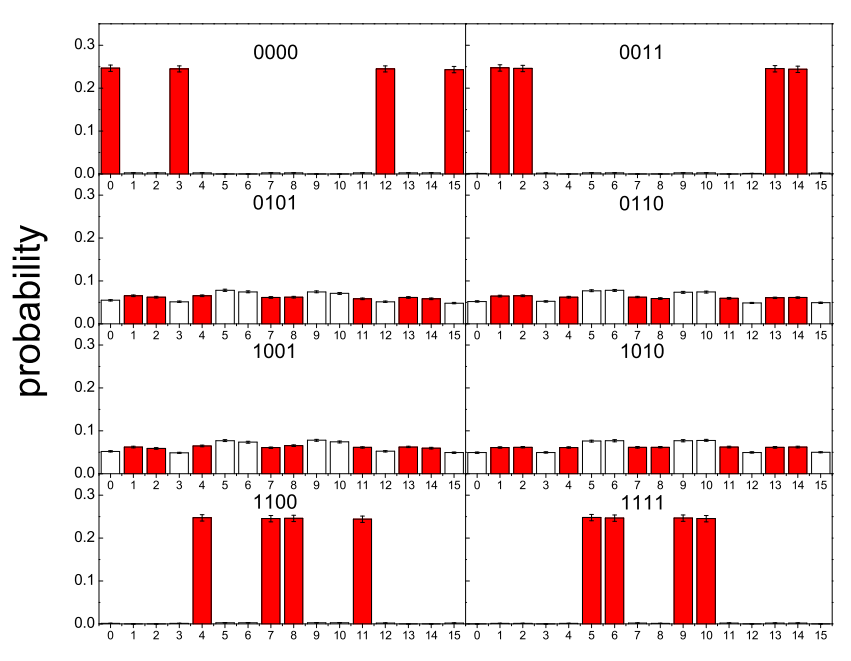

FIG. 4: (Color on line). Probability distribution for the fourparty QCCS when the four parties share two identical EPR states.

the parties to get the correct value of $F$ is $75 \%$ in this case.

In experiment, The EPR state is generated with 100 $\mathrm{mW}$ UV pulses. We manipulate $(\mathrm{A}, \mathrm{B})$ and $(\mathrm{C}, \mathrm{D})$ independently with twofold coincidences and combine their results to get the probability distribution for the fourparty QCCS.

Fig. 4 shows the experimental result when these four parties share two EPR states. The average success probability we obtain is $(73.89 \pm 1.33) \%$. Compared to the experimental result of the entangled state we have prepared, we can see that it is the genuine four-photon entangled part $(|G H Z\rangle$ in Eq. (3)) making the success probability reach $(81.54 \pm 1.38) \%$.

In conclusion, we have prepared a four-photon entangled state with a visibility as high as $(95.35 \pm 0.45) \%$. By using this state to realize a four-party QCCS, we prove that the state we have prepared violates a kind of Bell's inequality indirectly. This four-photon state can be used to fulfill decoherence-free quantum information processing [16] and other advanced quantum communication schemes.

This work was funded by National Natural Science Foundation of China.
[1] D. M. Greenberger, M. A. Horne, A. Shimony, and A. Zeilinger, Am. J. Phys. 58, 1131 (1990).

[2] W. Tittel, J. Brendel, H. Zbinden, and N. Gisin, Phys. Rev. Lett. 84, 4737 (2000); T. Jennewein, C. Simon, G. Weihs, H. Weinfurter, and A. Zeilinger, Phys. Rev. Lett. 84, 4729 (2000); D. S. Naik, C. G. Peterson, A. G. White, A. J. Berglund, and P. G. Kwiat, Phy. Rev. Lett. 84, 4733 (2000).

[3] D. Deutsch and R. Jozsa, Proc. R. Soc. London, Ser. A 439, 553 (1992).

[4] K. Mattle, H. Weinfurter, P. G. Kwiat, and A. Zeilinger, Phys. Rev. Lett. 76, 4656 (1996).

[5] C. H. Bennett, G. Brassard, C. Crépeau, R. Jozsa, A. Peres, and W. K. Wootters, Phys. Rev. Lett. 70, 1895 (1993); D. Bouwmeester, J.-W. Pan, K. Mattle, M. Eibl, H. Weinfurter, and A. Zeilinger, Nature (London) 390, 575 (1997).

[6] R. Cleve and H. Buhrman, Phy. Rev. A 56, 1201 (1997).

[7] P. Xue, Y.-F Huang, Y.-S Zhang, C.-F Li, and G.-C Guo, Phy. Rev. A 64, 032304 (2001).

[8] P. Xue, C.-F. Li, Y.-S Zhang, and G.-C Guo, J. Opt. B: Quantum Semiclassical Opt. 3, 219 (2001).
[9] J.-W. Pan, M. Daniell, S. Gasparoni, G. Weihs, and A. Zeilinger, Phy. Rev. Lett. 86, 4435 (2001).

[10] M. Eibl, S. Gaertner, M. Bourennane, C. Kurtsiefer, M. Żukowski, and H. Weinfurter, Phy. Rev. Lett. 90, 200403 (2003).

[11] N. Kiesel, C. Schmid, U. Weber, G. Tóth, O. Gühne, R. Ursin, and H. Weinfurter, Phy. Rev. Lett. 95, 210502 (2005).

[12] P. G. Kwiat, K. Mattle, H. Weinfurter, A. Zeilinger, A. V. Sergienko, and Y. Shih, Phys. Rev. Lett. 75, 4337 (1995).

[13] Č. Brukner, M. Żukowski, J.-W Pan, and A. Zeilinger, Phys. Rev. Lett. 92, 127901 (2004).

[14] P. G. Kwiat, E. Waks, A. G. White, I. Appelbaum, and P. H. Eberhard, Phys. Rev. A 60, R773 (1999).

[15] H. Weinfurter and M. Żukowski, Phys. Rev. A 64, 010102(R) (2001).

[16] M. Bourennane, M. Eibl, S. Gaertner, C. Kurtsiefer, A. Cabello, and H. Weinfurter, Phys. Rev. Lett. 92, 107901 (2004). 